\title{
Towards Reviving Electroweak Baryogenesis with a Fourth Generation
}

\author{
Wei-Shu Hou and Masaya Kohda \\ Department of Physics, National Taiwan University, Taipei 10617, Taiwan \\ Correspondence should be addressed to Masaya Kohda; mkohda@hep1.phys.ntu.edu.tw
}

Received 22 June 2012; Accepted 27 December 2012

Academic Editor: Tao Han

Copyright ( 2013 W.-S. Hou and M. Kohda. This is an open access article distributed under the Creative Commons Attribution License, which permits unrestricted use, distribution, and reproduction in any medium, provided the original work is properly cited.

\begin{abstract}
Electroweak baryogenesis is an attractive scenario for explaining the baryon asymmetry of the universe. However, it does not work within the standard model due to two reasons: (1) the strength of CP violation from the Kobayashi-Maskawa mechanism with three generations is too small; (2) the electroweak phase transition is not first order for the experimentally allowed Higgs boson mass. We discuss possibilities to solve these problems by introducing a fourth generation of fermions and how electroweak baryogenesis might be revived. We also discuss briefly the recent observation of a Higgs-like boson with mass around $125 \mathrm{GeV}$, which puts the fourth generation in a difficult situation, and the possible way out.
\end{abstract}

\section{Introduction}

The origin of the baryon asymmetry of the universe (BAU) is not only a big mystery in particle physics and cosmology, but it is a core problem related to our very existence. The observational evidence is very compelling. There is no indication for any macroscopic objects made from antiparticles. The antiparticles observed in cosmic rays are thought to be of secondary origin. Thus, it is accepted that the universe contains negligible amount of antibaryons, as compared to the baryons that constitute usual matter (including ourselves!), or $n_{\bar{b}} / n_{b} \ll 1$. The standard big bang cosmology requires the baryon-to-entropy ratio of the universe to be [1]

$$
\frac{n_{B}}{s}=\frac{n_{b}-n_{\bar{b}}}{s} \simeq 0.9 \times 10^{-10}
$$

If one does not take the view that this is merely an initial condition for the expanding universe, then the challenge is not so much the dominance of radiation, but that whether there is any matter left from the primordial matter-antimatter annihilation.

Many scenarios have been attempted to explain the origins of the minute amount of leftover matter based on a dynamical mechanism (baryogenesis). A common basis for such mechanisms is provided by the three Sakharov conditions [2,3], which consist of (i) baryon number (B) violation, (ii) $C$ and $\mathrm{CP}$ violation, and (iii) departure from equilibrium. In order to produce the BAU, these three conditions have to be satisfied simultaneously at some stage in the early universe.

Among many proposals, electroweak baryogenesis (EWBG) [4] is one of the well-motivated and appealing mechanisms to explain BAU, especially since its ingredients can be possibly probed by the Large Hadron Collider (LHC). This mechanism could operate during the electroweak phase transition (EWPT), occurring when the temperature of the universe is around $T_{c} \sim 100 \mathrm{GeV}$, and it is a possible baryogenesis mechanism within the standard model (SM) of particle physics. One should recall the truly remarkable fact that the SM carries all the necessary ingredients for the Sakharov conditions.

(1) $\mathbf{B}$ is violated due to the chiral anomaly induced by the $S U(2) \times U(1)$ gauge interaction. B-violating processes are nonperturbative phenomena mediated by topologically nontrivial SU(2) gauge field configurations. Although the instanton-mediated $\mathbf{B}$ violation at zero temperature is highly suppressed, the rate of $\mathbf{B}$ violation at finite temperature is not negligible via the 
so-called sphaleron transition, especially when the temperature is around or above $T_{c}$.

(2) $C$ is violated by the $V-A$ structure of the weak interaction, while CP is violated by the KobayashiMaskawa (KM) mechanism [5] with three generations of quarks. A complex phase in the CabibboKobayashi-Maskawa (CKM) matrix provides the source of CP violation, which nicely describes all terrestrially observed CP-violating phenomena, that is, $\mathrm{CP}$ violation in the $K$ - and $B$-meson (even the $D$ meson) systems.

(3) $\mathrm{SU}(2) \times \mathrm{U}(1)$ gauge symmetry is expected to be restored at high temperature above $T_{c}$. Therefore, as the universe cools down to a temperature of around $T_{c}$, the universe undergoes a phase transition (the EWPT) from symmetric phase of $\mathrm{SU}(2) \times \mathrm{U}(1)$ to the broken phase. If the EWPT is first order, the phase transition proceeds through bubble nucleation of the broken phase, where expansion of the bubbles fills up the entire universe. Thus, temporal departure from equilibrium is achieved near the surface of the expanding bubble.

Unfortunately, the measured parameters of the SM appear to be insufficient to bring about EWBG. The SM fails to explain the BAU due to two reasons. The first reason is that the KM mechanism with three quark generations cannot offer large enough $\mathrm{CP}$ violation $(\mathrm{CPV})$ to produce the observed baryon-to-entropy ratio during the EWPT. The second reason is that the EWPT is not first order for a Higgs boson with $m_{h}>114 \mathrm{GeV}$, required already by direct search at the LEP [6] and now indicated to be around $125 \mathrm{GeV}$ at the LHC $[7,8]$. Therefore, physics beyond the SM is needed for EWBG, and various extensions of the SM have been investigated from this point of view. In particular, there are extensive studies (for a recent review on EWBG, see [9]; and references therein) for the two Higgs doublet model and supersymmetric extensions of the SM, such as the MSSM and the NMSSM. It should be noted, however, that MSSM itself is under stress from the LHC, and its case for EWBG is only marginal [9]. One of the biggest means of attraction to have EWBG is that the implied new physics should be accessible at the LHC, at least partially.

In this contribution, we consider a four-generation (4G) extension of the SM and discuss a possibility to revive EWBG (beside a possible revival of EWBG, the $4 \mathrm{G}$ has another interesting implication on baryogenesis, if it is long-lived; see [10]) fully within the SM framework, as $4 G$ does not introduce anything that was not already contained in SM. We focus on the quark sector, which includes fourth generation quarks $t^{\prime}$ and $b^{\prime}$. Compared to the three-generation case, the $4 \mathrm{G}$ quarks bring three additional mixing angles and two additional complex phases in the $4 \times 4$ CKM matrix, thus providing new sources of $\mathrm{CP}$ violation. One can argue intuitively for a possible effect of new $\mathrm{CP}$ violation on BAU by studying basis-independent invariants constructed from the quark mass matrices (or the Yukawa coupling matrices), which are extensions of the Jarlskog determinant in the SM. One of us [11] pointed out that such an invariant quantity can be highly enhanced compared to the three-generation case, mainly due to the large masses (or the large Yukawa couplings) of $t^{\prime}$ and $b^{\prime}$ (see $[12,13]$ for an earlier discussion on 4G-enhanced CP violation for BAU). As for the issue of the EWPT, a one-loop analysis shows that the $4 \mathrm{G}$ quarks do not play a positive role towards a first-order phase transition [14]. However, given that bounds on the $4 \mathrm{G}$ quark masses from direct search at the LHC have entered $600 \mathrm{GeV}$ level $[15,16]$, which is beyond the tree-level perturbative unitarity bound (UB) of $500-550 \mathrm{GeV}$ [17], we have to reconsider the problem of EWPT beyond perturbation theory. In this regard, the nature of the EWPT with $4 \mathrm{G}$ quarks is still quite an open problem (for earlier works which address the issue of the EWPT within the four-generation framework (but not within the SM framework), see $[14,18]$ (supersymmetric model with the $4 \mathrm{G}$ fermions) and [19] (dynamical electroweak symmetry breaking model due to strong four-fermion interactions among the $4 \mathrm{G}$ fermions)).

The recent observation of a Higgs-like object with mass around $125 \mathrm{GeV}$ poses a special impasse situation, since a naive application of heavy $t^{\prime}$ and $b^{\prime}$ quarks in the loop would enhance the gluon-gluon fusion production of the Higgs boson by about an order of magnitude, which is not supported by $g g \rightarrow V V$ data $[7,8]$, where $V$ is the $W$ or $Z$ boson. Furthermore, quadratic corrections to the Higgs mass from heavy $t^{\prime}$ and $b^{\prime}$ quarks make the light Higgs mass rather difficult to sustain. With the deep conflict between a light Higgs and having 4G quarks beyond UB, it may seem cavalier to relegate this again to nonperturbative treatments. However, the aim of this work is very modest, which is just to show the nontrivial nature of $4 \mathrm{G}$ towards EWBG. Since the source of CPV arises from off-diagonal couplings, the Higgs boson does not directly enter EWBG computation. We will discuss towards the end the possibility that the observed $125 \mathrm{GeV}$ boson could be a dilaton, rather than a bona fide SM Higgs boson, or it may be a pseudo-Goldstone Higgs boson.

This paper is organized as follows. In Section 2, we discuss the effect of $\mathrm{CP}$ violation from the fourth generation quark sector on BAU. Extending the EWBG mechanism in the case of the SM to the fourth generation case, we estimate the amount of the BAU produced during a first-order EWPT. In Section 3, we summarize the EWPT in the SM briefly and discuss possible mechanisms to induce a first-order phase transition within the four-generation framework. Finally, in Section 4, we offer some discussions and give our summary.

\section{CP Violation}

One of the reasons for the failure of EWBG based on SM is the insufficiency of CP violation from the KM phase. This situation can be naively understood as follows.

In the SM with three generations, a basis-independent invariant for CP violation is given by the well-known Jarlskog determinant [20],

$$
\left.\operatorname{det}\left[M_{u} M_{u}^{\dagger}, M_{d} M_{d}^{\dagger}\right]\right|_{3-\text { gen. }} \equiv i J_{\mathrm{SM}}
$$


where $M_{u, d}$ are the up- and down-type quark mass matrices. In terms of quark masses and CKM matrix elements, one gets

$$
\begin{aligned}
J_{\mathrm{SM}}= & 2 \mathscr{J}_{\mathrm{SM}}\left(m_{t}^{2}-m_{c}^{2}\right)\left(m_{t}^{2}-m_{u}^{2}\right)\left(m_{c}^{2}-m_{u}^{2}\right) \\
& \times\left(m_{b}^{2}-m_{s}^{2}\right)\left(m_{b}^{2}-m_{d}^{2}\right)\left(m_{s}^{2}-m_{d}^{2}\right),
\end{aligned}
$$

where $\mathscr{F}_{\mathrm{SM}}$ is twice the area of any triangle defined by the unitarity condition of the CKM matrix, $V^{\dagger} V=\mathbf{1}$, with the experimentally determined value of $\mathscr{J}_{\mathrm{SM}} \simeq 3 \times 10^{-5}$. One can estimate a naive strength of $\mathrm{CP}$ violation during the EWPT by constructing a dimensionless quantity from $J_{\mathrm{SM}}$ and the critical temperature, $T_{c} \sim 100 \mathrm{GeV}$. This gives $J_{\mathrm{SM}} / T_{c}^{12} \sim 10^{-19}$, which is too small to account for BAU in (1).

It is readily observed that the smallness of $J_{\mathrm{SM}}$ originates mainly from the powers of light quark masses, $m_{c}^{2} m_{b}^{4} m_{s}^{2} / T_{c}^{8} \sim$ $10^{-15}$, with milder suppression from the CKM factor $\mathscr{J}_{\mathrm{SM}}$. Motivated by this observation and a possible hint of new physics in $b \rightarrow s$ transition, it was suggested in [11] to introduce the $4 \mathrm{G}$ quarks and replace (3) by an analogous quantity involving second to fourth generation quarks (or equivalently, treating the first two generations as degenerate),

$$
\begin{aligned}
J_{(2,3,4)}^{s b}= & 2 \mathscr{J}_{s}\left(m_{t^{\prime}}^{2}-m_{t}^{2}\right)\left(m_{t^{\prime}}^{2}-m_{c}^{2}\right)\left(m_{t}^{2}-m_{c}^{2}\right) \\
& \times\left(m_{b^{\prime}}^{2}-m_{b}^{2}\right)\left(m_{b^{\prime}}^{2}-m_{s}^{2}\right)\left(m_{b}^{2}-m_{s}^{2}\right) \\
\sim & \frac{\mathscr{J}_{s}}{\mathscr{J}_{\mathrm{SM}}}\left(\frac{m_{t^{\prime}}^{2}}{m_{t}^{2}}-1\right) \frac{m_{t^{\prime}}^{2}}{m_{c}^{2}} \frac{m_{b^{\prime}}^{4}}{m_{b}^{2} m_{s}^{2}} J_{\mathrm{SM}},
\end{aligned}
$$

where $\mathscr{J}_{s}=\operatorname{Im}\left(V_{t s}^{*} V_{t b} V_{t^{\prime} b}^{*} V_{t^{\prime} s}\right)$. In fact, $J_{(2,3,4)}^{s b}$ is a leading term of a basis-independent set of invariants for CP violation in the four-generation case and is given by $\operatorname{Tr}\left[M_{u} M_{u}^{\dagger}, M_{d} M_{d}^{\dagger}\right]^{3}$, up to an overall factor $[20,21]$. Due to heaviness of $t^{\prime}$ and $b^{\prime}$, $J_{(2,3,4)}^{s b}$ can be highly enhanced compared to $J_{\mathrm{SM}}$ in (3). For $m_{t^{\prime}} \sim m_{b^{\prime}} \sim 600 \mathrm{GeV}$, for instance, the enhancement factor reaches $\sim 10^{18}$ solely from the mass factors, thus, seemingly possible to overcome the smallness of $J_{\mathrm{SM}}$.

In the following, after we briefly review EWBG in the SM case, we take one step further and examine the previous expectation of $4 \mathrm{G}$-enhanced $\mathrm{CP}$ violation for the EWBG scenario.

A concrete mechanism of EWBG within SM was proposed by Farrar and Shaposhnikov (FS) [22-25]. In the FS mechanism, a baryon asymmetry is produced through CP violating scattering of quarks at the surface of an expanding bubble, that is, the bubble wall generated during a first-order EWPT. CP asymmetry in reflection of the quarks off the bubble wall is induced by thermal effects, that is, interactions of the quarks with the $W$ and charged-Higgs bosons in the cosmic plasma. FS included these effects employing a quasiparticle picture and treated the scattering problem in a quantum mechanical manner. Solving an effective Dirac equation for the quasiparticles, they found that $\mathrm{CP}$ violation from the KM phase is sufficient to explain the BAU under optimal conditions, contrary to the naive argument given previous.
The very attractive SM explanation for BAU by FS was, however, refuted by subsequent works by Gavela et al. [2628 ]. These authors pointed out that the width of the quasiparticle (damping rate) was not included in the study of FS, and they found that inclusion of the width reduces the baryon asymmetry to a negligible amount. This result was also confirmed by Huet and Sather (HS) [29], who interpreted the damping as quantum decoherence phenomenon induced by plasma effects, leading to reduction of the CP asymmetry, in which quantum mechanical coherence plays an essential role. After the demonstration of these results, it was (re)accepted that $\mathrm{CP}$ violation from the $\mathrm{KM}$ phase is not sufficient to explain BAU. This conclusion is in accordance with the naive dimensional argument of $J_{\mathrm{SM}}$ given previous. We now estimate, by extending the work of HS, the baryon asymmetry generated by the FS mechanism in the four-generation case.

We assume the existence of a first-order phase transition. The baryon asymmetry generated during the first-order EWPT is given by [29]

$$
\frac{n_{B}}{s} \sim-\frac{10^{-2}}{T} \int \frac{d \omega}{2 \pi} n_{0}(\omega)\left[1-n_{0}(\omega)\right] \frac{\Delta \mathbf{p} \cdot \mathbf{v}_{W}}{T} \Delta(\omega),
$$

where $n_{0}(\omega)=1 /[\exp (\omega / T)+1]$ is the Fermi-Dirac distribution, $\Delta \mathbf{p} \equiv \mathbf{p}_{L}-\mathbf{p}_{R}$ is the difference between lefthanded and right-handed quasiparticle momenta for a given energy $\omega$, and $\mathbf{v}_{W}$ is the velocity of the expanding bubble wall. We neglect $\mathcal{O}\left(\mathbf{v}_{W}^{2}\right)$ contribution by assuming $\left|\mathbf{v}_{W}\right| \sim 0.1$. We take $T \sim T_{c} \sim 100 \mathrm{GeV}$ in the previous formula. $\Delta(\omega)$ is a reflection asymmetry defined by

$$
\Delta(\omega) \equiv \operatorname{Tr}\left[\bar{R}_{L R}^{\dagger} \bar{R}_{L R}-R_{L R}^{\dagger} R_{L R}\right]
$$

where reflection coefficients $R_{L R}$ and $\bar{R}_{L R}$ are matrices in flavor space; that is, $R_{L R}^{f i}$ is the reflection coefficient for $q_{L}^{i} \rightarrow q_{R}^{f}$, where $i$ and $f$ refer to quark flavors, and $\bar{R}_{L R}^{f i}$ corresponds to the CP-conjugate process. $\Delta(\omega)$ provides the $\mathrm{CP}$ asymmetry for the reflection rate of a left-handed quasiparticle, incident from the symmetric phase with an energy $\omega$, summed over all flavors. We further assume that the EWPT is strongly first order, so that the generated baryon asymmetry is not washed out by the sphaleron processes in the broke phase (see next section). We return to discuss the issue of order of phase transition in $4 \mathrm{G}$ context later.

$\Delta(\omega)$ is obtained by solving an effective Dirac equation for the quasiparticles in the presence of space-dependent quark mass terms. Assuming planar wall with zero thickness, the effective Dirac equation is given by

$$
\begin{array}{cc}
\left(\begin{array}{cc}
2\left(\omega-\Omega_{L}+i \gamma+\frac{1}{3} i \sigma \cdot \partial\right) & M_{u, d} \theta(z) \\
M_{u, d}^{\dagger} \theta(z) & 2\left(\omega-\Omega_{R}+i \gamma-\frac{1}{3} i \sigma \cdot \partial\right)
\end{array}\right) \\
\times \Psi_{u, d}(z)=0,
\end{array}
$$

where $\Psi_{u}=\left(\psi_{u}, \psi_{c}, \psi_{t}, \psi_{t^{\prime}}\right)^{T}, \Psi_{d}=\left(\psi_{d}, \psi_{s}, \psi_{b}, \psi_{b^{\prime}}\right)^{T}$, and each $\psi_{i}$ are four-component spinor wavefunctions. In (7), 
$\Omega_{L(R)}$ is a thermal mass matrix for the left- (right-) handed quasiparticles, $\gamma$ is the quasiparticle width, and we use the one calculated in QCD [30], $\gamma \simeq 0.15 g_{s}^{2} T$, neglecting possible flavor-dependent corrections from the Yukawa interactions.

Following HS, we solve the effective Dirac equations analytically based on the Green's function method, which gives perturbative expansions of the reflection coefficients in terms of the quark mass matrices $M_{u, d}$. The leading-order contribution to $\Delta(\omega)$ from the scattering of the $d$-type quarks is given by

$$
\begin{aligned}
\Delta_{d}(\omega)= & \frac{4}{3}\left(\frac{27 \pi \alpha_{W} T^{2}}{64 \Omega_{0} M_{W}^{2}}\right)^{3}\left[1+\left(\frac{\omega-\Omega_{0}}{\gamma}\right)^{2}\right]^{-6} \\
& \times\left(\frac{1}{6 \gamma}\right)^{9} \operatorname{Im} \operatorname{Tr}\left[M_{u} M_{u}^{\dagger}, M_{d} M_{d}^{\dagger}\right]^{3},
\end{aligned}
$$

where $\Omega_{0} \simeq g_{s} T / \sqrt{6}$ is the dominant part in the thermal masses of quarks, coming from QCD. The $4 \mathrm{G}$ effects enter solely through the last factor in (8), which arises as one follows the scattering of an incoming $d$-type quark against the bubble wall. In the three-generation case, this factor is nothing but the Jarlskog determinant, discussed previous,

$$
\begin{aligned}
\operatorname{Im} & \left.\operatorname{Tr}\left[M_{u} M_{u}^{\dagger}, M_{d} M_{d}^{\dagger}\right]^{3}\right|_{3-\text { gen. }} \\
& =\left.3 \operatorname{Im} \operatorname{det}\left[M_{u} M_{u}^{\dagger}, M_{d} M_{d}^{\dagger}\right]\right|_{3-\text { gen }} \\
& =3 J_{\mathrm{SM}},
\end{aligned}
$$

where $J_{S M}$ is defined in (2) and (3). On the other hand, the four-generation counterpart is more complicated, but it can be simplified [21] by exploiting the hierarchical structure of the quark masses and the CKM matrix elements, with a mild assumption on new mixings, $\left|V_{t d} V_{t^{\prime} d}^{*}\right| \ll\left|V_{t i} V_{t^{\prime} i}^{*}\right|\left(i=s, b, b^{\prime}\right)$. Then, the last factor in (8) is approximated as

$$
\begin{aligned}
\operatorname{Im} \operatorname{Tr} & {\left[M_{u} M_{u}^{\dagger}, M_{d} M_{d}^{\dagger}\right]^{3} } \\
& \simeq-6 \mathscr{J}_{s}\left(m_{t^{\prime}}^{2}-m_{t}^{2}\right) m_{t^{\prime}}^{2} m_{t}^{2} m_{b^{\prime}}^{4} m_{b}^{2},
\end{aligned}
$$

where $\mathscr{J}_{s}=\operatorname{Im}\left(V_{t s}^{*} V_{t^{\prime} b}^{*} V_{t b} V_{t^{\prime} s}\right)$. One notes that $\operatorname{Im} \operatorname{Tr}\left[M_{u} M_{u}^{\dagger}, M_{d} M_{d}^{\dagger}\right]^{3} \simeq-3 J_{(2,3,4)}^{s b}$, where $J_{(2,3,4)}^{s b}$ is defined in (4). Therefore, $\Delta_{d}(\omega)$ can be actually enhanced by large masses of $t^{\prime}$ and $b^{\prime}$ compared to the SM case, as anticipated in [11].

As discussed by HS, dimensionless perturbative expansion parameters in the Green's function method are given by $M_{u, d} /(6 \gamma) \simeq M_{u, d} /(1.4 T)$, which are of order unity or larger for the $t, t^{\prime}$, and $b^{\prime}$ quarks, given $T \sim 100 \mathrm{GeV}$ during the EWPT. Hence, the perturbative expansion breaks down for these heavy quarks, and the previous result for $\Delta_{d}(\omega)$ could be reduced due to large $m_{b^{\prime}}$ (equation (8) is obtained by the expansion in the $d$-type quark mass matrix $M_{d}$ based on the Green's function method. The $u$-type quark mass matrix $M_{u}$ in (8) originates from the thermal mass matrix for the $d$ type quasiparticles, that is, the Yukawa interaction with the plasma. Thus, the heaviness of only the $b^{\prime}$ quark matters to
$\Delta d(\omega))$ if the perturbation is not used. Thus, the previous result might overestimate the $\mathrm{CP}$ asymmetry from the $d$-type quarks. The $u$-type quark contribution, at leading order, is the same as (8) except for the difference of the overall sign, hence, leading to a complete cancelation at this level. The previous mentioned remark is, however, also applied for the $u$-type quark contribution, and a possible amount of reduction would be even larger as it contains the contributions from the two heavy quarks, $t$ and $t^{\prime}$. We naively accept (8) as a dominant contribution to the reflection asymmetry $\Delta(\omega)$, simply neglecting the $u$-type quark contribution (there is another type of major contribution to $\Delta(\omega)$, called $\Delta_{7}$ in HS, which arises when the finite quark masses in the broken phase are taken into account in the self-energy of quarks. While $\Delta_{7}$ gives larger contribution to the BAU than (8) in the threegeneration case, we confirmed that the contribution from (8) dominates in the four-generation case for experimentally allowed masses of $4 \mathrm{G}$ quarks).

Eventually, the baryon asymmetry generated during the EWPT is given by

$$
\frac{n_{B}}{s} \sim 0.9 \times 10^{-10}\left(\frac{\mathcal{J}_{s}}{10^{-4}}\right)\left(\frac{m_{t^{\prime}}}{650 \mathrm{GeV}}\right)^{4}\left(\frac{m_{b^{\prime}}}{650 \mathrm{GeV}}\right)^{4},
$$

where $T=100 \mathrm{GeV}$ and $M_{W}(T)=50 \mathrm{GeV}$ are adopted. $\mathscr{F}_{s}$ includes new CKM parameter $V_{t^{\prime} s} V_{t^{\prime} b}^{*}$ which can be measured via flavor observables related with $b \rightarrow s$ transition. We note that measurements for the CP violating phase $\phi_{s}$ in $B_{s}-\bar{B}_{s}$ mixing, the forward-backward asymmetry for $B \rightarrow$ $K^{*} \mu^{+} \mu^{-}$, and the $B_{s} \rightarrow \mu^{+} \mu^{-}$rate are making rapid progress at the LHC. Theoretical predictions for these observables are not subject to hadronic uncertainties so much; hence, they are useful to constrain $V_{t^{\prime} s} V_{t^{\prime} b}^{*}$ [31]. From the recent LHC data, $\mathscr{J}_{s}$ of around $10^{-4}$ with $m_{t^{\prime}}=650 \mathrm{GeV}$ seems to be at the border of the experimentally allowed region [32]. However, with rising $m_{t^{\prime}}$ (and $m_{b^{\prime}}$ ), there is a tendency that $\mathscr{J}_{s}$ drops, but the high powers of $m_{t^{\prime}} m_{b^{\prime}}$ in (11) should easily compensate for it.

With reasonable $4 \mathrm{G}$ parameters, therefore, the FS mechanism with $4 \mathrm{G}$ quarks seems to generate the correct order of magnitude of the baryon-to-entropy ratio and, hence, can in principle explain BAU. We note that the previous estimate of BAU relies on the perturbative treatments of the Yukawa couplings/masses of the quarks including $t^{\prime}$ and $b^{\prime}$ in a few steps, while the LHC bounds on the $4 \mathrm{G}$ quark masses suggest a nonperturbative nature of the corresponding Yukawa couplings. For instance, the Green's function method invokes the perturbation in the quark mass matrices as we already discussed. Besides this point, the thermal properties for the quasiparticles are obtained via perturbative calculations; for example, the thermal mass matrices are evaluated at oneloop level. Therefore, our result would be semiquantitative at most. Nevertheless, given remarkable enhancement of the $\mathrm{CP}$ asymmetry due to the $4 \mathrm{G}$ quarks and the agreement with the observed BAU as shown in (11), our finding should deserve further investigation. We further remark that the CPviolating Jarlskog-like invariants of (2) and (10) are of purely algebraic nature. Thus, we suspect that a fully dynamical calculation should still reflect this fact. 


\section{Electroweak Phase Transition}

Besides CP violation, the other essential issue for EWBG is the nature of the EWPT. EWBG requires first-order EWPT, which proceeds through nucleation and growth of brokenphase bubbles. In addition, there is a stronger requirement $[12,13]$ on the EWPT as explained later.

In EWBG, the baryon asymmetry is generated at the EWPT utilizing the B-violating sphaleron transition. However, the sphaleron transition must decouple just after the phase transition, otherwise the generated baryon asymmetry would be washed out. In the broken phase, the sphaleron transition rate is proportional to the Boltzmann factor $\exp \left(-E_{\mathrm{sph}} / T\right)$, where $E_{\mathrm{sph}}$ is the energy of a sphaleron configuration and is given by $E_{\mathrm{sph}} \sim 4 \pi \phi / \mathrm{g}$, where $\phi$ is the thermal average of the Higgs field, $\left\langle H^{0}\right\rangle_{T}=\phi / \sqrt{2}$, and $g$ is the $\mathrm{SU}(2)$ gauge coupling. In order to ensure the decoupling of the sphaleron transition in the broken phase, $\phi$ should be large enough to suppress the previous rate, leading to the condition $\phi_{c} / T_{c} \gtrsim 1$, where $\phi_{c}$ is given by $\left\langle H^{0}\right\rangle_{T_{c}}=\phi_{c} / \sqrt{2}$. Therefore, the first-order EWPT has to be strong enough to avoid a washout of the generated baryon asymmetry.

A basic tool to analyze the EWPT is the finite temperature effective potential (FTEP) ([33-35], and for a review on EWPT and FTEP, see [36] and references therein). In SM, the one-loop FTEP is given by

$$
V^{(0)}(\phi)=V_{0}(\phi)+V_{1}^{(0)}(\phi)+V_{1}^{(T)}(\phi, T),
$$

where $V_{0}$ is the tree-level potential for the Higgs field, $V_{1}^{(0)}$ is the temperature independent one-loop contribution, and $V_{1}^{(T)}(\phi, T)$ represents the finite temperature correction to the zero-temperature potential. $V_{1}^{(0)}$ in Landau gauge and $\overline{\mathrm{MS}}$ scheme is given by the Coleman-Weinberg potential

$$
V_{1}^{(0)}(\phi)=\sum_{i=h, \chi, W, Z, t} n_{i} \frac{m_{i}^{4}(\phi)}{64 \pi^{2}}\left[\ln \frac{m_{i}^{2}(\phi)}{\mu^{2}}-C_{i}\right],
$$

where $m_{i}^{2}(\phi)$ is the $\phi$-dependent squared mass for particle $i$ with $n_{i}$ the corresponding number of degrees of freedom. See Table 1 for the definitions, where we note that fermions contribute with opposite sign.

The one-loop finite temperature contribution, $V_{1}^{(T)}$, is given by

$$
\begin{aligned}
& V_{1}^{(T)}(\phi, T) \\
& \quad=\frac{T^{4}}{2 \pi^{2}}\left[\sum_{i=h, \chi, W, Z} n_{i} J_{B}\left[\frac{m_{i}^{2}(\phi)}{T^{2}}\right]+n_{t} J_{F}\left[\frac{m_{t}^{2}(\phi)}{T^{2}}\right]\right],
\end{aligned}
$$

where $J_{B}$ and $J_{F}$ are defined by

$$
\begin{aligned}
& J_{B}(a)=\int_{0}^{\infty} d x x^{2} \ln \left[1-e^{-\sqrt{x^{2}+a}}\right], \\
& J_{F}(a)=\int_{0}^{\infty} d x x^{2} \ln \left[1+e^{-\sqrt{x^{2}+a}}\right] .
\end{aligned}
$$

TABLE 1: The $\phi$-dependent squared masses $m_{i}^{2}(\phi)$ and the number of degrees of freedom $n_{i}$ for particle $i$ in SM. $m_{i}^{2}(\phi=v)$ gives the physical masses for $v=246 \mathrm{GeV}$, which is the vacuum expectation value of the Higgs field. $C_{i}$ are given for $\overline{\mathrm{MS}}$ scheme. We include the contributions coming from the Higgs boson $h$, the would-be Nambu-Goldstone bosons $\chi$, the gauge bosons $W$ and $Z$, and the top quark $t$.

\begin{tabular}{lcccc}
\hline Particle & $m_{i}^{2}(\phi)$ & $m_{i}^{2}(v)$ & $n_{i}$ & $C_{i}$ \\
\hline$h$ & $-m^{2}+3 \lambda \phi^{2}$ & $2 \lambda v^{2}$ & 1 & $3 / 2$ \\
$\chi$ & $-m^{2}+\lambda \phi^{2}$ & 0 & 3 & $3 / 2$ \\
$W$ & $\frac{g^{2}}{4} \phi^{2}$ & $\frac{g^{2}}{4} v^{2}$ & 6 & $5 / 6$ \\
$Z$ & $\frac{g^{2}+g^{\prime 2}}{4} \phi^{2}$ & $\frac{g^{2}+g^{\prime 2}}{4} v^{2}$ & 3 & $5 / 6$ \\
$t$ & $\frac{\lambda_{t}^{2}}{2} \phi^{2}$ & $\frac{\lambda_{t}^{2}}{2} v^{2}$ & -12 & $3 / 2$ \\
\hline
\end{tabular}

In the high temperature limit where $m(\phi) \ll T, J_{B, F}$ can be expanded in terms of $m(\phi) / T$ (high-temperature expansion) as

$$
\begin{aligned}
J_{B}\left(\frac{m^{2}}{T^{2}}\right)= & -\frac{\pi^{4}}{45}+\frac{\pi^{2}}{12} \frac{m^{2}}{T^{2}}-\frac{\pi}{6}\left(\frac{m^{2}}{T^{2}}\right)^{3 / 2} \\
& -\frac{1}{32} \frac{m^{4}}{T^{4}} \ln \frac{m^{2}}{a_{b} T^{2}}+\mathcal{O}\left(\frac{m^{6}}{T^{6}}\right), \\
J_{F}\left(\frac{m^{2}}{T^{2}}\right)= & \frac{7 \pi^{4}}{360}-\frac{\pi^{2}}{24} \frac{m^{2}}{T^{2}} \\
& -\frac{1}{32} \frac{m^{4}}{T^{4}} \ln \frac{m^{2}}{a_{f} T^{2}}+\mathcal{O}\left(\frac{m^{6}}{T^{6}}\right),
\end{aligned}
$$

where $a_{b}=16 \pi^{2} \exp \left(3 / 2-2 \gamma_{E}\right)$, and $a_{f}=\pi^{2} \exp \left(3 / 2-2 \gamma_{E}\right)$, that is, $\ln a_{b} \approx 5.4076$ and $\ln a_{f} \approx 2.6351$.

Using high temperature expansion, the one-loop FTEP can be written as

$$
V(\phi, T) \simeq D\left(T^{2}-T_{o}^{2}\right) \phi^{2}-E T \phi^{3}+\frac{\lambda(T)}{4} \phi^{4},
$$

where

$$
\begin{gathered}
D=\frac{2 m_{W}^{2}+m_{Z}^{2}+2 m_{t}^{2}}{8 v^{2}}, \\
T_{o}^{2}=\frac{m_{h}^{2}}{4 D}, \\
E=\frac{2 m_{W}^{3}+m_{Z}^{3}}{4 \pi v^{3}}, \\
\lambda(T)=\lambda-\frac{3}{16 \pi^{2} v^{4}} \quad \\
\times\left(2 m_{W}^{4} \ln \frac{\mu^{2}}{A_{B} T^{2}}+m_{Z}^{4} \ln \frac{\mu^{2}}{A_{B} T^{2}}-4 m_{t}^{4} \ln \frac{\mu^{2}}{A_{F} T^{2}}\right) .
\end{gathered}
$$




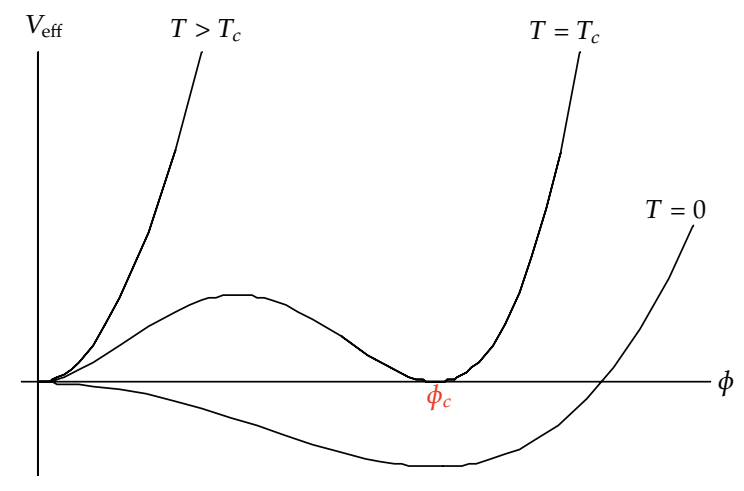

FIGURE 1: Typical forms of the effective potential in a case of the firstorder phase transition for $T=0, T=T_{c}$, and $T>T_{1}$.

Here, $m_{i}^{2} \equiv m_{i}^{2}(v)$ with $v=246 \mathrm{GeV}$ are physical masses for particle $i$, and $\ln A_{B}=\ln a_{b}-3 / 2$, as well as $\ln A_{F}=$ $\ln a_{f}-3 / 2$. In (17), terms which do not have $\phi$ dependence are omitted. For sake of illustration, we also neglect contributions from $h$ and $\chi$ by assuming that $h$ is lighter than $W$ and $Z$, though this is phenomenologically not correct. One sees that only weak bosons contribute to the coefficient $E$ of the cubic term in (17); hence, $E$ is rather small in SM.

With this FTEP, one can examine the behavior of the EWPT analytically. For $T>T_{1} \equiv$ $\sqrt{8 \lambda\left(T_{1}\right) D T_{o}^{2} /\left(8 \lambda\left(T_{1}\right) D-9 E^{2}\right)}$, the only minimum of the effective potential, (17), is $\phi=0$. Hence, the electroweak symmetry is restored. As the electroweak symmetry is broken at $T=0$, the phase transition must occur at a temperature $T$ that satisfies $T_{1}>T>0$. Inspection of (17), one clearly sees that the phase transition is first order for $E \neq 0$, taking the form depicted in Figure 1, and is second order for $E=0$. When $E \neq 0$, the critical temperature $T_{c}$ and $\phi_{c}$ are given by

$$
T_{c}^{2}=\frac{\lambda\left(T_{c}\right) D T_{0}^{2}}{\lambda\left(T_{c}\right) D-E^{2}}, \quad \phi_{c}=\frac{2 E T_{c}}{\lambda\left(T_{c}\right)} .
$$

Then, the strength of the first-order phase transition is given by

$$
\frac{\phi_{c}}{T_{c}}=\frac{2 E}{\lambda\left(T_{c}\right)} \sim \frac{4 E v^{2}}{m_{h}^{2}} .
$$

One notes that a lighter Higgs boson is preferred to maintain a stronger first-order phase transition.

At high temperature, a certain class of higher-order diagrams, the so-called ring diagrams (or daisy diagrams) [36], give significant contributions to the FTEP. Dominant contributions from the ring-diagrams can be resummed, and it amounts to shifting the masses $m_{i}^{2}(\phi)$ for bosons in the one-loop contributions, given in (13) and (14), to thermal masses given by $\mathscr{M}_{i}^{2}(\phi, T) \equiv m_{i}^{2}(\phi)+\Pi_{i}(T)$, where $\Pi_{i}(T)$ is the one-loop self-energy of particle $i$ in the infrared limit. As for gauge bosons, only the longitudinal modes receive corrections to the masses when one-loop self-energies are adopted for $\Pi_{i}(T)$. Basically, this forbids the longitudinal modes of $W$ and $Z$ to contribute to the cubic term in (17).
As a consequence, the coefficient of the cubic term is reduced as

$$
E=\frac{2 m_{W}^{3}+m_{Z}^{3}}{4 \pi v^{3}} \longrightarrow \frac{2}{3} \frac{2 m_{W}^{3}+m_{Z}^{3}}{4 \pi v^{3}} \sim 9.5 \times 10^{-3},
$$

leading to the reduction of the strength of the EWPT.

With these results, the sphaleron decoupling condition, $\phi_{c} / T_{c} \gtrsim 1$, reads

$$
m_{h} \lesssim \sqrt{4 E} v \sim 42 \mathrm{GeV} .
$$

However, this condition conflicts with the mass bound from direct search at the LEP, $m_{h}>114 \mathrm{GeV}$ [6]. Furthermore, recent results from the LHC indicate a Higgs-like particle at $125 \mathrm{GeV}$. So, the EWPT is not strongly first order in the SM, and the baryon asymmetry generated during EWPT cannot be retained in the broken phase.

For a heavier Higgs boson, $m_{h} \gtrsim m_{W}$, the approximations used in the previous analysis are no longer correct in the SM. In particular, higher-order diagrams beyond the ring diagrams also become nonnegligible at critical temperature, and a nonperturbative analysis is required. Lattice studies [37-40] suggest, however, that there is an endpoint of firstorder EWPT around $m_{h} \sim 70 \mathrm{GeV}$, above which the transition turns into a continuous crossover. Therefore, there is no EWPT for the experimentally preferred Higgs boson mass.

For successful EWBG, then, physics beyond the SM is also required. It is clear that the existence of the cubic term in the FTEP of (17) is essential for a first-order phase transition, and the cubic term arises from $\left(m^{2}\right)^{3 / 2}$ term in the high temperature expansion for the bosonic loop function $J_{B}$, given in (16), while there is no such term from fermionic contributions. So, if a new boson with strong coupling to the Higgs boson is introduced, their thermal loop can enhance the cubic term, and the EWPT can be strengthened. On the other hand, introducing a new fermion does not affect the cubic term and, hence, does not improve the strength of the EWPT at one-loop level.

Based on the one-loop result, therefore, the introduction of $4 \mathrm{G}$ fermions seems useless from the viewpoint of making a first-order EWPT. However, given that bounds on the $4 \mathrm{G}$ quark masses from direct search at the LHC have reached beyond the $600 \mathrm{GeV}$ level, that is, beyond the perturbative unitarity bound of $500-550 \mathrm{GeV}$, we have to reconsider the problem of the EWPT beyond perturbative level. Interestingly, several studies (for zero temperature case) suggest that strong Yukawa couplings of $4 \mathrm{G}$ quarks can induce new bound states of $4 \mathrm{G}$ quarks [41-43]. If such Yukawa bound states are bound tightly enough so that they do not dissolve around the critical temperature of the EWPT, bosonic bound states may contribute to the FTEP via loop effect and may induce the cubic term, leading to strongly first-order phase transition. Beside this possibility, if the $4 \mathrm{G}$ quarks $Q$ form a pair condensate, $\langle\bar{Q} Q\rangle \neq 0$, due to strong Yukawa couplings, an effective description of the theory would be given by a three Higgs doublet model (1 elementary +2 composite Higgs doublet) [41] (the EWPT in multicomposite Higgs doublet model with the $4 \mathrm{G}$ fermions was studied in [19], where 
the composite Higgs fields appear as bound states of the $4 \mathrm{G}$ fermions, formed by strong four-fermion interactions). Therefore, the potential for the Higgs field would be modified even at tree level, and dynamics of the EWPT could be drastically changed.

\section{Discussion and Summary}

Let us continue the discussion from the previous section. Bosonization is only one aspect of very heavy $4 \mathrm{G}$ quarks that could change the landscape for EWBG. After all, the bosonization described previous is due to strong Yukawa coupling. A special feature of the SM is that fermion, in fact all, masses reflect a dynamical coupling. But fermions are special in that there is no theory of these Yukawa couplings, and, neglecting neutrinos, they span a range of six orders of magnitude! If a fourth generation exists above the unitarity bound, the strong Yukawa coupling could induce $\langle\bar{Q} Q\rangle$ condensation, which in principle could replace the usual condensation of the Higgs field as the electroweak symmetry breaking mechanism. A "bootstrap" dynamical symmetry breaking (DSB) equation was recently formulated [44] and studied [45], and it was found numerically that DSB can occur for Yukawa coupling $\lambda_{Q} \gtrsim 4 \pi$. For such strong Yukawa coupling, our traditional notions for EWBG may have to be reconsidered.

The difficulty for the bootstrap DSB scenario is again the newly observed $[7,8] 125 \mathrm{GeV}$ boson at the LHC. However, the observed state could be a dilaton of scale invariance violation, rather than the genuine SM Higgs boson. The couplings of the dilaton to vector bosons and fermions are suppressed by $v / f$ compared to the SM Higgs boson case, where $f$ is the dilaton decay constant, while $\gamma \gamma$ and $g g$ couplings of the dilaton are essentially free parameters depending on the details of scale invariance violation [46]. Therefore, if the observed signal arises mostly from the gluon fusion, the dilaton could mimic the SM Higgs boson. Discrimination is provided by the detection of the Higgs production through the vector boson fusion (VBF), or the bremsstrahlung off a vector boson $(\mathrm{VH})$. In particular, important modes are the VBF-produced $\gamma \gamma$ mode and the $b \bar{b}$ and $\tau \bar{\tau}$ modes in this regard. As VBF and $\mathrm{VH}$ production are subdominant compared with gluon fusion, these modes are not yet firmly established by the LHC experiments. It is interesting that the bootstrap DSB equation is scale invariant by construction, and at the present level of study, the scale is introduced heuristically as a physical condition for the bootstrap [45]; hence, a dilaton is in principle allowed. Whether the $125 \mathrm{GeV}$ state is the SM-like Higgs or a dilaton with rather modified couplings can be checked by ATLAS and CMS.

Even if the $125 \mathrm{GeV}$ object is verified as SM-like Higgs boson, the strong Yukawa coupling of $4 \mathrm{G}$ quarks may still be relevant. The lightness of the Higgs boson may be because it is a pseudo-Goldstone boson from a $\mathrm{TeV}$ scale strongly coupled theory, which indeed the previous $\lambda_{Q} \gtrsim 4 \pi$ situation seems to qualify it as a candidate. Pseudo-Goldstone Baryogenesis (PGBG) has been advocated [47] as a possible mechanism, where strong coupling brings about parametric enhancement of effective dimension-six interactions that loosen the relation between Higgs self-coupling and the Higgs mass; it is $\lambda\left(T_{c}\right)$ that really appears in (20), and Higgs mass enters only through the standard relation of $m_{h}^{2}=2 \lambda v^{2}$. Thus, whether one could have PGBG at work or not has to be tested by checking the Higgs boson self-coupling, which likely can be done only at an $e^{+} e^{-}$Linear Collider with energy above $500 \mathrm{GeV}$, which will take several decades.

Of course, the formulation of the bootstrap DSB equation is not yet at the level to demonstrate a possible PGBG, and much more work needs to be done. The formulation of the bootstrap DSB equation itself may offer a different path towards the study of order of phase transition. The equation is a coupled set of two integral equations of loop momentum in the ladder approximation [45]. At finite temperature, these would become four equations, since the temporal integration would be replaced by a summation. One could check the temperature dependence of DSB, both in finding the critical $T_{c}$ when symmetry is restored, but also check what is " $\phi_{c}$," the critical Higgs field expectation value, and whether its strength allows a strongly first-order transition. Of course, this is not yet done, but it may offer further insight that is along a different path than the usual approach of [36] discussed in the previous section.

To summarize, EWBG is an attractive scenario to address the BAU puzzle, especially because this scenario is based on particle physics models that can be tested at the LHC. Although the EWBG scenario fails within the minimal SM with three quark generations, introduction of the fourth generation may revive the scenario, offering hope to solve the problems in the SM case. CP violation coming from the $4 \times 4$ CKM matrix would be highly enhanced compared to the SM, mainly through the large masses (or the large Yukawa couplings) of $t^{\prime}$ and $b^{\prime}$ quarks. Naive extension of the EWBG mechanism of SM to the $4 \mathrm{G}$ case shows that this $\mathrm{CP}$ violating effect can be large enough to explain BAU with reasonable $4 \mathrm{G}$ parameters. The issue of whether the EWPT becomes strongly first order with $4 \mathrm{G}$ quarks is still an open problem, especially since the bounds on $4 \mathrm{G}$ quark masses from direct search at the LHC have reached the $600 \mathrm{GeV}$ level, which is beyond the perturbative unitarity bound of $500-550 \mathrm{GeV}$. A mechanism of the strongly first-order phase transition may be accommodated by new bound states and/or $\bar{Q} Q$ condensation of $4 \mathrm{G}$ quarks, both induced by strong Yukawa couplings, which could modify the FTEP at loop/tree level. Higgs boson search at the LHC has uncovered a new boson with SM Higgs features. The study is still ongoing, and its nature is important to obtain better understanding for the EWPT. All in all, the possibility of providing both sufficient $\mathrm{CP}$ violation as well as perhaps a strongly first-order phase transition (which we did not demonstrate), all rooted in large Yukawa couplings of fourth generation quarks, makes this a worthy pursuit.

\section{Acknowledgments}

The authors are grateful to Y. Kikukawa for collaboration on the FS mechanism in the four-generation case, presented in 
Section 2. M. Kohda is supported by the NTU Grant no. 10R40044 and the Laurel Program, and W.-S. Hou by the Academic Summit Grant NSC 100-2745-M-002-002-ASP of the National Science Council of Taiwan and various NTU grants under the Excellence Program of the Ministry of Education of Taiwan.

\section{References}

[1] J. Beringer, J. F. Arguin, R. M. Barnett et al., "Review of particle physics," Physical Review D, vol. 86, Article ID 010001, 2012.

[2] A. D. Sakharov, "CP symmetry violation, C-asymmetry and baryonic asymmetry of the universe," Zhurnal Eksperimentalno i Teoreticheskoi Fiziki, Pisma, vol. 5, p. 32, 1967.

[3] A. D. Sakharov, "CP symmetry violation, C-asymmetry and baryonic asymmetry of the universe," Journal of Experimental and Theoretical Physics Letters, vol. 5, p. 24, 1967.

[4] V. A. Kuzmin, V. A. Rubakov, and M. E. Shaposhnikov, "On anomalous electroweak baryon-number non-conservation in the early universe," Physics Letters B, vol. 155, no. 1-2, pp. 36-42, 1985.

[5] M. Kobayashi and T. Maskawa, "CP-Violation in the renormalizable theory of weak interaction," Progress of Theoretical Physics, vol. 49, no. 2, pp. 652-657, 1973.

[6] R. Barate, R. Bruneliere, I. de Bonis et al., "Search for the standard model higgs boson at LEP," Physics Letters B, vol. 565, pp. 61-75, 2003.

[7] G. Aad, T. Abajyan, B. Abbott et al., "Observation of a new particle in the search for the Standard Model Higgs boson with the ATLAS detector at the LHC," Physics Letters B, vol. 716, no. 1, pp. 1-29, 2012.

[8] S. Chatrchyan, V. Khachatryan, A. M. Sirunyan et al., "Observation of a new boson at a mass of $125 \mathrm{GeV}$ with the CMS experiment at the LHC," Physics Letters B, vol. 716, no. 1, pp. 30-61, 2012.

[9] D. E. Morrissey and M. J. Ramsey-Musolf, "Electroweak baryogenesis," New Journal of Physics, vol. 14, Article ID 125003, 2012.

[10] H. Murayama, V. Rentala, J. Shu, and T. T. Yanagida, "Saving fourth generation and baryon number by living long," Physics Letters B, vol. 705, no. 3, pp. 208-211, 2011.

[11] W. S. Hou, "Source of CP violation for baryon asymmetry of the universe," Chinese Journal of Physics, vol. 47, p. 134, 2009.

[12] M. E. Shaposhnikov, "Possible appearance of the baryon asymmetry of the universe in an electroweak theory," Jounal of Experimental and Theoretical Physics Letters, vol. 44, no. 8, pp. 465-468, 1986.

[13] M.E. Shaposhnikov, "Baryon asymmetry of the universe in standard electroweak theory," Nuclear Physics B, vol. 287, pp. 757-775, 1987.

[14] R. Fok and G. D. Kribs, "Four generations, the electroweak phase transition, and supersymmetry," Physical Review D, vol. 78, no. 7, Article ID 075023, 2008.

[15] S. Chatrchyan, V. Khachatryan, A. M. Sirunyan et al., "Search for heavy, top-like quarkpair production in the dilepton final state in $p p$ collisions at sqrt(s) $=7 \mathrm{TeV}$,' Physics Letters B, vol. 716, pp. 103-121, 2012.

[16] S. Chatrchyan, V. Khachatryan, A. M. Sirunyan et al., "Search for heavy bottom-like quarks in $4.9 \mathrm{fb}^{-1}$ of pp collisions at $\sqrt{\mathrm{s}}=7$ TeV," Journal of High Energy Physics, vol. 1205, p. 123, 2012.
[17] M. S. Chanowitz, M. A. Furman, and I. Hinchliffe, "Weak interactions of ultra heavy fermions," Physics Letters B, vol. 78, no. 2-3, pp. 285-289, 1978.

[18] S. W. Ham, S. K. Oh, and D. Son, "Electroweak phase transition in the minimal supersymmetric standard model with four generations," Physical Review D, vol. 71, Article ID 015001, 6 pages, 2005.

[19] Y. Kikukawa, M. Kohda, and J. Yasuda, “The strongly coupled fourth family and a first-order electroweak phase transition. Iquark sector," Progress of Theoretical Physics, vol. 122, no. 2, pp. 401-426, 2009.

[20] C. Jarlskog, "Flavor projection operators and applications to CP violation with any number of families," Physical Review D, vol. 36, no. 7, pp. 2128-2136, 1987.

[21] W. S. Hou, Y. Y. Mao, and C. H. Shen, "Leading effect of CP violation with four generations," Physical Review D, vol. 82, Article ID 036005, 10 pages, 2010.

[22] G. R. Farrar and M. E. Shaposhnikov, "Baryon asymmetry of the universe in the minimal standard model," Physical Review Letters, vol. 70, no. 19, pp. 2833-2836, 1993.

[23] G. R. Farrar and M. E. Shaposhnikov, "Erratum 'Baryon asymmetry of the universe in the minimal standard model," Physical Review Letters, vol. 71, p. 210, 1993.

[24] G. R. Farrar and M. E. Shaposhnikov, "Baryon asymmetry of the universe in the standard electroweak theory," Physical Review D, vol. 50, no. 2, pp. 774-818, 1994.

[25] G. R. Farrar and M. E. Shaposhnikov, "Note added to 'Baryon asymmetry of the universe in the standard model," http://arxiv.org/abs/hep-ph/9406387.

[26] M. B. Gavela, P. Hernandez, J. Orloff, and O. Pene, "Standard model Cp-violation and baryon asymmetry," Modern Physics Letters A, vol. 9, no. 9, p. 795, 1994.

[27] M. B. Gavela, M. Lozano, J. Orloff, and O. Pène, "Standard model CP-violation and baryon asymmetry (I). Zero temperature," Nuclear Physics B, vol. 430, no. 2, pp. 345-381, 1994.

[28] M. B. Gavela, P. Hernandez, J. Orloff, O. Pène, and C. Quimbay, "Standard model CP-violation and baryon asymmetry (II). Finite temperature," Nuclear Physics B, vol. 430, no. 2, pp. 382426, 1994.

[29] P. Huet and E. Sather, "Electroweak baryogenesis and standard model CP violation," Physical Review D, vol. 51, no. 2, pp. 379394, 1995.

[30] E. Braaten and R. D. Pisarski, "Calculation of the quark damping rate in hot QCD," Physical Review D, vol. 46, no. 4, pp. 18291834, 1992.

[31] W. S. Hou, M. Kohda, and F. Xu, "Measuring the fourthgeneration $\mathrm{b} \rightarrow \mathrm{s}$ quadrangle at the LHC," Physical Review D, vol. 84, no. 9, Article ID 094027, 7 pages, 2011.

[32] W. S. Hou, M. Kohda, and F. Xu, "Hints for a low $B_{s} \rightarrow \mu^{+} \mu^{-}$ rate and the fourth generation," Physical Review D, vol. 85, no. 9, Article ID 097502, 5 pages, 2012.

[33] L. Dolan and R. Jackiw, "Symmetry behavior at finite temperature," Physical Review D, vol. 9, no. 12, pp. 3320-3341, 1974.

[34] S. Weinberg, "Gauge and global symmetries at high temperature," Physical Review D, vol. 9, no. 12, pp. 3357-3378, 1974.

[35] G. W. Anderson and L. J. Hall, "Electroweak phase transition and baryogenesis," Physical Review D, vol. 45, no. 8, pp. 26852698, 1992.

[36] M. Quiros, "Finite temperature fieldtheory and phase transitions," http://arxiv.org/abs/hep-ph/9901312. 
[37] M. Gürtler, E. M. Ilgenfritz, and A. Schiller, "Where the electroweak phase transition ends," Physical Review D, vol. 56, no. 7, pp. 3888-3895, 1997.

[38] M. Laine and K. Rummukainen, "What's new with the electroweak phase transition?" Nuclear Physics B, vol. 73, no. 1-3, pp. 180-185, 1999.

[39] F. Csikor, Z. Fodor, and J. Heitger, "End point of the hot electroweak phase transition," Physical Review Letters, vol. 82, no. 1, pp. 21-24, 1999.

[40] Y. Aoki, F. Csikor, Z. Fodor, and A. Ukawa, “The end point of the first-order phase transition of the $\mathrm{SU}(2)$ gauge-Higgs model on a 4-dimensional isotropic lattice," Physical Review D, vol. 60, no. 1, Article ID 013001, pp. 1-8, 1999.

[41] P. Q. Hung and C. Xiong, "Dynamical electroweak symmetry breaking with a heavy fourth generation," Nuclear Physics B, vol. 848, no. 2, pp. 288-302, 2011.

[42] K. Ishiwata and M. B. Wise, "Fourth generation bound states," Physical Review D, vol. 83, no. 7, Article ID 074015, 8 pages, 2011.

[43] T. Enkhbat, W. S. Hou, and H. Yokoya, "Early LHC phenomenology of Yukawa-bound heavy $\bar{Q}$ mesons," Physical Review D, vol. 84, no. 9, Article ID 094013, 14 pages, 2011.

[44] W. S. Hou, "Some unfinished thoughts on strong yukawa couplings," Chinese Journal of Physics, vol. 50, p. 375, 2012.

[45] Y. Mimura, W. S. Hou, and H. Kohyama, "Bootstrap dynamical symmetrybreaking with new heavy chiral quarks," http://arxiv.org/abs/1206.6063.

[46] D. Elander and M. Piai, "The decay constant of the holographic techni-dilaton and the $125 \mathrm{GeV}$ boson," Nuclear Physics B, vol. 867, no. 3, pp. 779-809, 2013.

[47] B. Grinstein and M. Trott, "Electroweak baryogenesis with a pseudo-Goldstone Higgs boson," Physical Review D, vol. 78, no. 7, Article ID 075022, 28 pages, 2008. 

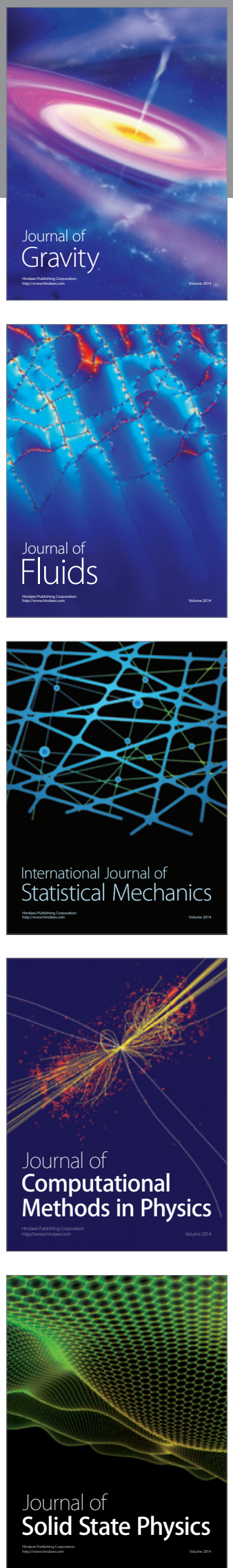

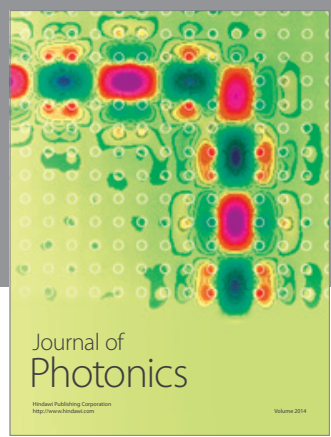

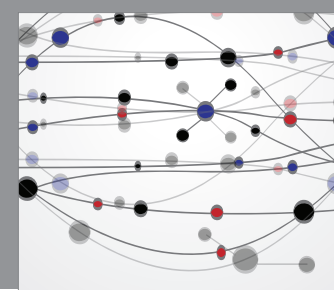

The Scientific World Journal

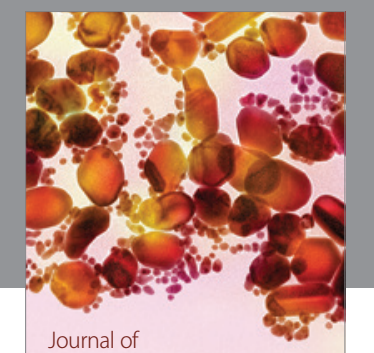

Soft Matter
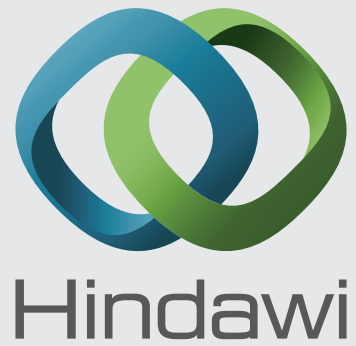

Submit your manuscripts at

http://www.hindawi.com
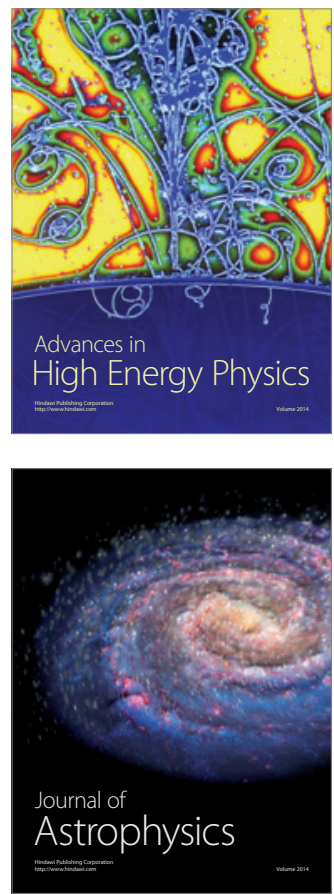
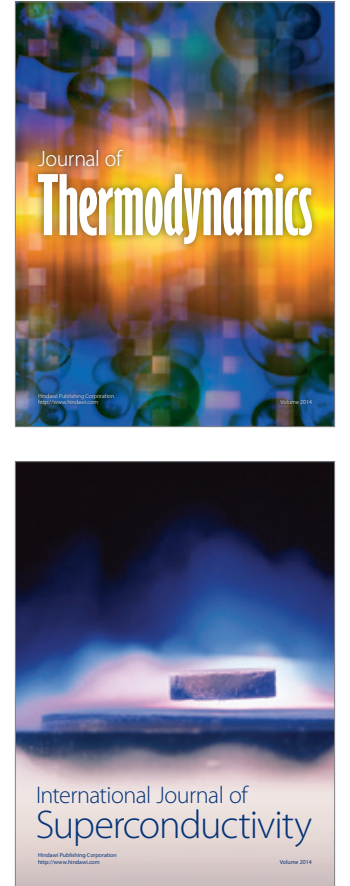
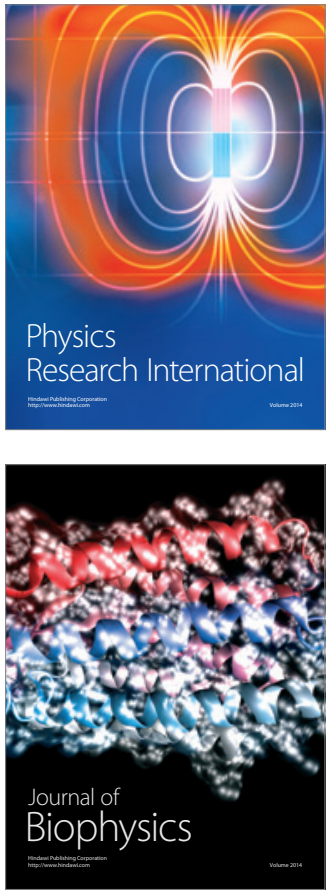
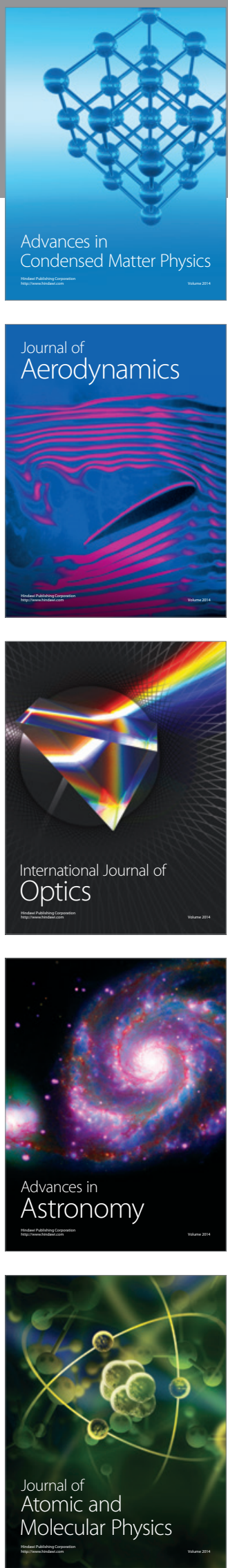